\title{
101 THINGS THAT YOU MIGHT HAVE WONDERED ABOUT SPACE PLASMA WAVE RESEARCH BUT WERE AFRAID TO ASK!
}

\author{
R. R. Anderson*
}

\begin{abstract}
Whistlers are electromagnetic waves generated by lightning discharges traveling through the ionosphere where the dispersion relations show that the higher frequency emissions travel faster than the low frequency emissions. Satellite analog wave receivers allowed the Very Low Frequency (VLF) (less than $30 \mathrm{kHz}$ ) waves to be transmitted to the ground by modulating a Very High Frequency carrier. Spectrograms of some whistlers showed emissions with rising components beginning below the ion cyclotron frequencies and later referred to as proton, helium, or oxygen ion whistlers. Analyses of the crossover frequency, the asymptotic frequency, and the dampening rate yielded relative mass density, magnetic field magnitude, and electron temperature. The INJUN V satellite instrumentation was able to determine the propagation direction up or down the geomagnetic field line. Whistlers verified the calibrations. VLF saucers centered on regions of intense energetic electron precipitation were shown to be upgoing from the auroral region. The upper frequency limits of the filter banks on these satellites were $35 \mathrm{kHz}$ and $100 \mathrm{kHz}$. Subsequent satellite receivers progressively increased the upper frequency limits of both the digital filter banks and analog wideband receivers. The dominant auroral emission was auroral kilometric radiation (AKR) whose intensity peaks around 250 $\mathrm{kHz}$. AKR observations from the UC Berkeley FAST spacecraft show that it is primarily generated slightly above the local relativistic electron cyclotron frequency. AKR frequency limits extend from below $15 \mathrm{kHz}$ to at least $800 \mathrm{kHz}$ showing that it is generated over a large spatial range of several Earth radii. High resolution wave forms show tones and emissions embedded in the AKR data indicating significant ion influences. Observations of Type II and Type III solar radio bursts produced by coronal mass emissions and solar flares provide details of the interplanetary medium density structure.
\end{abstract}

* Department of Physics and Astronomy, University of Iowa, Iowa City, IA 52242, USA 